\title{
Joint modeling of a linear mixed effects model for self- esteem from mean ages 13 to 22 and a generalized linear model for anxiety disorder at mean age 33
}

\author{
Henian Chen ${ }^{1 *}$, Yangxin Huang ${ }^{1}$ and Nanhua Zhang ${ }^{2}$ \\ *Correspondence: hchen1@health.usf.edu

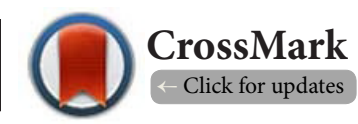 \\ 'Department of Epidemiology \& Biostatistics, College of Public Health, University of South Florida, Tampa, FL, USA. \\ 2Division of Biostatistics and Epidemiology, Cincinnati Children's Hospital Medical Center, Cincinnati, OH, USA.
}

\begin{abstract}
Recent work has identified the transition from adolescence to young adulthood as a period with distinct characteristics that is important for understanding of human development. Self-esteem plays a critical role in this developmental process. We use self-esteem data measured at mean ages 13, 16 and 22, and anxiety disorder diagnosis at mean age of 33 to examine the impact of development of self-esteem on onset of adult anxiety disorder. To analyze these data, we propose a Bayesian joint model with: (1) a linear mixed effects model for the longitudinal measurements, and (2) a generalized linear model for the binary primary endpoint. Our analysis indicates that the mean level of self-esteem, not the change of self-esteem, significantly predicts the onset of adult anxiety disorder. A comparison shows that the joint model yields better predictive accuracy than a two-step model. The respective area under ROC curve (AUC) is 0.60 and 0.75 for the two-step model and the joint model, respectively. The two-step estimate may be biased because this method ignores variability in the individual random effects. We conclude that joint model is the most advantageous model to analyze early life longitudinal data combined with later binary outcome.
\end{abstract}

Keywords: Joint model, two-step model, anxiety disorder, self-esteem

\section{Introduction}

Recent theoretical and empirical work has identified the transition from adolescence to young adulthood as a period with distinct characteristics and importance for the understanding of human development [1]. Self-esteem plays a critical role in this developmental process. Studies on self-esteem development from adolescence to young adulthood have found moderate increases during adolescence and slower increases during young adulthood [2], while other studies report that self-esteem declines during adolescence, partially explained by adolescent concerns with self-image and related issues associated with puberty, but increases gradually throughout adulthood [3]. From a theoretical standpoint, changes in self-esteem coincide with major life events or transitions [4]. Nevertheless, there is little agreement regarding the development of self-esteem through young adulthood due to few longitudinal-based studies conducted on a non-clinical adolescent population $[2,5]$.

Anxiety disorder, the most common one in all the mental disorders, may be associated with poor self-esteem [6]. Studies $[7,8]$ have found that subjects with anxiety disorders had lower levels of self-esteem, compared with non-clinical controls. However, no research has been found examining the impact of self-esteem development from adolescence to young adulthood on onset of adult anxiety disorder. Most of studies on relationship between self-esteem and anxiety disorder are limited to current self-esteem or the history of self-esteem at one time point. Moreover, clinical researchers are interested in how the development of self-esteem in early life impact adult anxiety disorder. The goal of the analysis is to provide an estimated probability of having anxiety disorder given individual profile of self-esteem over time. The longitudinal measurements of self-esteem are subject to measurement error as most other self-reported data. Also, the self-esteem level for each subject changes over time and the patterns of change vary for different subjects. Conventional models that do not consider these two sources of variation usually produce biased results. In the present study, we are interested in the association between self-esteem measured at mean ages of $13,16,22$ and anxiety disorder measured at mean age of 33 .

\section{Methods}

A longitudinal study of change in self-esteem from mean ages 13 to 22 and anxiety disorder at mean age 33

This paper uses data from the Children in the Community $(\mathrm{CIC})$ 
study (http://nyspi.org/childcom), a randomly sampled mean age 5 cohort living in rural and urban households in 1975 [9]. The study sample is a representative cross-section of two upstate New York counties from 1975. Self-esteem was measured in 1983 (mean age of 13), 1986 (mean age of 16) and 1994 (mean age of 22). Four items indexed global self-esteem in each protocol: (1) I feel that I have a number of good qualities; (2) I feel that my life is very useful; (3) I am a useful person to have around; and (4) I feel I do not have much to be proud of (reversed). The items were rated from 1 (false) to 4 (true), and the internal consistency of the scale formed by summing them was 0.64 in adolescence and 0.69 in young adulthood [10]. Anxiety disorder at mean age 33 (in 2005) was assessed with the Structured Clinical Interview for DSM-IV Axis I Disorders [11]. To simplify our model, we will focus on self-esteem and anxiety disorder data and ignore other covariates such as gender, race, and family SES. Table 1 shows the raw data of this study. As we can see the mean level of self-esteem increases from 9.33 to 9.96 from age 13 to age 22 for the entire sample. Subjects $(n=85)$ with adult anxiety disorder had a lower level of self-esteem and increased more slowly than those $(n=432)$ without any mental disorder.

\section{Analysis issues in joint modeling of longitudinal data}

The early development of joint models was primarily motivated and used for longitudinal and survival data [12-19]. Joint models for longitudinal and survival data are particularly relevant to many cancer/AIDS clinical trials and observational studies in which longitudinal biomarkers may be highly associated with time to event. Joint models allow the simultaneous estimation of parameters from both the longitudinal and survival processes in one model, accounting for the association between processes through the incorporation of shared random effects [18]. When a relationship exists between the longitudinal and survival processes, more efficient and reliable estimates are obtained through the use of a joint model compared to more naive methods, such as a time-dependent Cox model [20]. Although joint models for longitudinal and time-to-event data are widely recognized for their gain in efficiency and reduction in bias achieved over naïve methods, to the best of our knowledge, relatively few studies have been conducted on joint models for longitudinal measurements and binary outcomes. A binary outcome is usually a downgrading from a time-to-event outcome and, therefore, less informative. In applications where the timing of the event is not the primary interest, the analysis of binary outcome may be appropriate. In the application of predicting anxiety disorder at mean age 33 , our primary interest is whether the participant was clinically diagnosed of adult anxiety disorder in 2005. Since the clinical diagnosis of adult anxiety disorder was only performed for each subject in 2005 , the information on the exact time of anxiety disorder onset is left-censored and unavailable for all participants. Therefore, there is not much gain in applying a model for timeto-event data and a model for binary outcome is appropriate. The remainder of the article is organized as follows. In Section 2, we investigate specific joint models which include (i) a linear mixed effects model for the longitudinal measurement process (self-esteem), and (ii) a generalized linear model for the binary primary endpoint (clinical diagnosis of anxiety disorder). These two models are linked through the random-effects that characterize the underlying individual-specific longitudinal processes. In Section 3, we investigate a naïve two-step model. In Section 4, we apply both joint model and two-step model to the motivating dataset and compare these two models. We conclude the article with discussion in Section 5.

\section{A bayesian joint model with two submodels}

For subject $i=1, \ldots, n$ and discrete time point $j=1, \ldots, K$, we define the following:

(1) $R_{i}$ denotes the binary primary endpoint (which is the binary indicator of anxiety disorder).

(2) $Y_{i}=\left(y_{i}, \ldots, y_{i k}\right)$ denotes the $(1 \times K)$ complete-data vector of continuous longitudinal measures (longitudinal selfesteem measures) for subject $i$.

A joint model under Bayesian framework can be hierarchically formulated as follows.

$$
\begin{aligned}
& Y_{i j}=\beta_{0 i}+\beta_{1 i} \times A g e+\varepsilon_{i j^{\prime}}, \varepsilon_{i j} \sim N\left(0, \sigma^{2}\right) \\
& \beta_{0 i}=\beta_{0}+b_{0 i^{\prime}} \beta_{1 i}=\beta_{1}+b_{1 i^{\prime}}\left(b_{o i^{\prime}} b_{1 i}\right)^{\top} \sim N_{2}(0, \Sigma) \\
& \text { Logit }\left(R_{i}\right)=a_{0}+a_{1} b_{0 i}+a_{2} b_{1 i}
\end{aligned}
$$

We specify prior distributions for all unknown parameters in the joint model as follows.

$$
\begin{gathered}
\beta_{k} \sim N(0,100)(k=0,1), a_{k} \sim N(0,100)(k=0,1,2,3) \\
\sigma^{-2} \sim \operatorname{Gamma}(0.01,0.01), \Sigma^{-1} \sim \text { Wishard }\left(I_{2^{\prime}} 3\right)
\end{gathered}
$$

We use Markov chain Monto Carlo (MCMC) technique via Gibbs sampler to estimate the unknown parameters simultaneously using publically-available WinBUGS software [21] and WinBUGS code is available upon requested from Authors. In particular,

Table 1. Self-esteem $($ Mean \pm SD) at different development stages $(\mathrm{N}=517)$.

\begin{tabular}{lllll}
\hline Mean age 33 & N & Mean age 13 & Mean age 16 & Mean age 22 \\
\hline Subjects with anxiety disorder & 85 & $8.9176(2.4212)$ & $8.7225(2.0201)$ & $9.3765(2.2677)$ \\
Healthy people without any mental disorder & 432 & $9.4129(2.0643)$ & $9.6005(1.9429)$ & $10.0786(1.7993)$ \\
Total & 517 & $9.3315(2.1325)$ & $9.4561(1.9808)$ & $9.9632(1.8998)$ \\
\hline
\end{tabular}


the MCMC scheme for drawing samples from the posterior distributions of all parameters is obtained by the Gibbs sampler. After collecting the final MCMC samples, we are able to draw statistical inference for the unknown parameters. See the articles [21,22] for detailed discussions of the Bayesian modeling approach and the implementation of the MCMC procedures, including the choice of the hyper-parameters, the iterative MCMC algorithm, sensitivity analysis and convergence diagnostics. When the MCMC implementation is applied to the actual data, convergence of the generated samples is assessed using standard tools within WinBUGS software. After convergence was achieved, one long chain was run which may be more efficient with the following considerations. We propose that, after an initial number of 10,000 burn-in iterations, every 10th MCMC sample is retained from the next 50,000 . Thus, we obtain 5,000 samples of targeted posterior distributions of the unknown parameters for statistical inference.

\section{Two-step model}

Another possible approach is a naïve two-step model [23-25]. Two-step model was first proposed by Tsiatis et al., [23] to analyze longitudinal and survival data. Some new development and application of the methods can be found in Ye et al. [25]. The first step estimates the individual random intercepts and slopes from a linear mixed-effects model using longitudinal self-esteem data:

$$
\begin{aligned}
& Y_{i j}=\beta_{0 i}+\beta_{1 i} \times A g e_{i j}+\varepsilon_{i j^{\prime}} \\
& \beta_{0 i}=\beta_{0}+b_{0 i^{\prime}} \beta_{1 i}=\beta_{1}+b_{1 i^{\prime}}
\end{aligned}
$$

The second step, the estimated values are incorporated into a logistic regression model with outcome measure of anxiety disorder:

$$
\operatorname{logit}\left(\mathrm{R}_{\mathrm{i}}\right)=\mathrm{a}_{0}+\mathrm{a}_{1} \mathrm{~b}_{0 \mathrm{i}}+\mathrm{a}_{2} \mathrm{~b}_{1 \mathrm{i}}
$$

Two-step model was widely used in mental health research. Perrin et al., [26] compared adults with and without schizophrenia on the linear slope of their height from birth to 2.5 years. Kasen et al., [27] used intercept and slope on earlier life stress from each subject to predict major depression at mean age of 60 using a logistic regression. Kokko et al., [28] used trajectories of prosocial behavior and physical aggression between 6 and 12 years of age to predict school dropout and physical violence at age 17 using logistic regression analysis. Piquero et al., [29] employed the developmental trajectories of criminal activity through age 40 to predict self-report measures of life failure at age 48. Dick et al., [30] used the probability of class membership in trajectories of externalizing behavior to test the effects of GABRA2 gene. Two-step model leads to potentially biased and inefficient estimates $[15,31]$. The intercepts and slopes from the linear mixed-effects model are fixed at the second step. The two-step model ignores the error inherent in the predicted random effects which are used as covariates in the logistic or linear regression model. Thus, the two-step method may produce biased estimates. We will compare these two models in next section.

\section{Data analysis and results}

To carry out the Bayesian inference, we specified the values of the hyper-parameters in the prior distributions. In the Bayesian analysis, we specified only the priors at the population level. We took weakly-informative prior distribution for the parameters in the joint models. In particular, (i) fixed-effects were taken to be independent normal distributions $N(0,100)$ for each component of the population parameter vectors $\alpha$ and $\beta$; (ii) for the variance parameters $\sigma^{2}$, we assume a limiting noninformative inverse gamma prior distribution, $\operatorname{IG}(0.01,0.01)$ so that the distribution has mean 1 and variance 100; (iii) the priors for the variance-covariance matrices of the randomeffects were taken to be inverse Wishart distributions $\operatorname{IW}\left(\Gamma_{1}\right.$, $3)$ and $\operatorname{IW}\left(\Gamma_{1}, 3\right)$ with covariance matrices $\Gamma_{1}=\Gamma_{2}=\operatorname{diag}(0.01$, $0.01)$. The MCMC sampler was implemented using WinBUGS for the two models.

Table 2 shows results from fitting the joint model and two-step model. As we can see that the two-step model gives smaller SE at the second step and narrower interval estimates than joint model. Results show that the mean level of self-esteem, not the change of self-esteem, significantly predicts the onset of adult anxiety disorder.

The Receiver Operating Characteristic (ROC) curves and the area under the ROC curve (AUC) for the joint model and two-step model are presented in Figure 1 (also see Table 2). The respective AUC are 0.60 for two-step model and 0.75 for the joint model. The AUC can be used as an overall estimate of its discriminating ability and sometimes is expressed as degree of accuracy. Greater AUC indicates higher accuracy. This finding suggests that the joint model improves sensitivity and specificity for predicting anxiety disorder.

\section{Discussion and conclusion}

Studies [6-8] have shown that subjects with anxiety disorders had lower levels of self-esteem, compared with non-clinical controls. This is the first longitudinal study to model the association between the development of self-esteem from mean ages 13 to 22 on anxiety disorder at mean age 33. We propose a Bayesian joint model to account for the binary primary endpoint (clinical diagnosis of adult anxiety disorder) and the longitudinal measurement (measures of self-esteem over a 9-year period). This model characterizes (i) within-subject patterns of longitudinal measurement, and (ii) the association between features of the longitudinal measurement process and the occurrence of the binary endpoint. In the Bayesian joint model proposed here, we specify the longitudinal response process as a standard linear mixed effects model, and hence the generalized linear model is linked to the longitudinal response process through the random intercept and slope. Results from the joint model indicate that the mean level 
Chen et al. Journal of Medical Statistics and Informatics 2015,

http://www.hoajonline.com/journals/pdf/2053-7662-3-1.pdf

Table 2. Two-step model and joint model.

\begin{tabular}{|c|c|c|c|c|c|c|c|c|}
\hline \multicolumn{4}{|c|}{ Two-step model } & \multicolumn{5}{|c|}{ Joint model } \\
\hline & Mean $(95 \% \mathrm{CI})$ & SE & OR $(95 \% \mathrm{CI})$ & AUC & Mean $(95 \% \mathrm{CI})$ & SE & OR $(95 \% \mathrm{CI})$ & AUC \\
\hline \multicolumn{9}{|c|}{ LME: Linear mixed-effects model } \\
\hline$\beta_{0}$ & $\begin{array}{l}9.5510 \\
(9.418,9.682)\end{array}$ & 0.0669 & -- & -- & $\begin{array}{l}9.5490 \\
(9.4180,9.6820)\end{array}$ & 0.0673 & -- & -- \\
\hline$\beta_{1}$ & $\begin{array}{l}0.0078 \\
(0.0535,0.1015)\end{array}$ & 0.0122 & -- & -- & $\begin{array}{l}0.0772 \\
(0.0533,0.1020)\end{array}$ & 0.0125 & -- & -- \\
\hline \multicolumn{9}{|c|}{ LR: Logistic regression } \\
\hline$\alpha_{0}$ & $\begin{array}{l}-1.6900 \\
(-1.9830,-1.4530)\end{array}$ & 0.1238 & $\begin{array}{l}0.1845 \\
(0.1377,0.2339)\end{array}$ & -- & $\begin{array}{l}-1.7760 \\
(-2.0809,-1.5040)\end{array}$ & 0.1505 & $\begin{array}{l}0.1693 \\
(0.1248,0.2222)\end{array}$ & -- \\
\hline$\alpha_{1}$ & $\begin{array}{l}-0.4145 \\
(-0.6551,-0.1700)\end{array}$ & 0.1238 & $\begin{array}{l}0.6607 \\
(0.5194,0.8437)\end{array}$ & -- & $\begin{array}{l}-0.4688 \\
(-0.7813,-0.1859)\end{array}$ & 0.1530 & $\begin{array}{l}0.6258 \\
(0.4578,0.8304)\end{array}$ & -- \\
\hline \multirow[t]{2}{*}{$\alpha_{2}$} & $\begin{array}{l}-2.0580 \\
(-4.4650,0.4043)\end{array}$ & 1.2410 & $\begin{array}{l}0.1277 \\
(0.0115,1.4983)\end{array}$ & -- & $\begin{array}{l}-2.6360 \\
(-5.9820,0.3244)\end{array}$ & 1.5910 & $\begin{array}{l}0.0716 \\
(0.0025,1.3832)\end{array}$ & -- \\
\hline & -- & -- & & 0.60 & -- & -- & & 0.75 \\
\hline
\end{tabular}

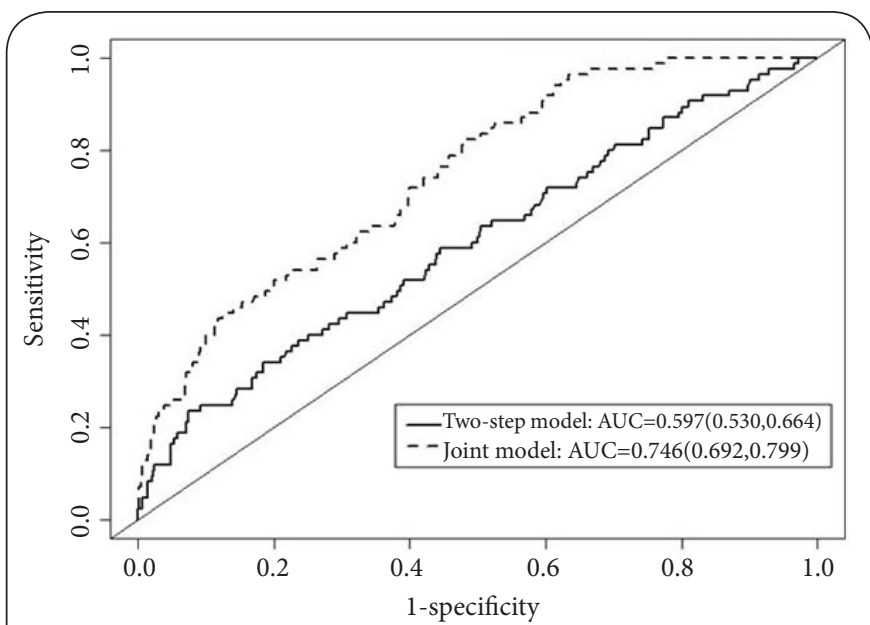

Figure 1. ROC curves with areas (AUC) and 95\% CI for joint model and two-step model.

of self-esteem, not the change of self-esteem, significantly predicts the onset of adult anxiety disorder.

Joint models for longitudinal and time-to-event data are ubiquitous and well-developed in the literature [32], joint models for longitudinal measurements and binary outcome are relatively rare. A binary outcome is usually a downgrading from a time-to-event outcome and therefore less informative. In applications where the timing of the event is not the primary interest, the analysis of binary outcome may be appropriate. In this paper, we also compared two different models, which are two-step model and joint model. The parameter estimates are similar for both the joint model and two-step model. However, the two-step model gives smaller SE and narrower interval estimates because the two-step model doesn't account for the error in the estimates of the random effects. The two-step model estimate may be biased because this method ignores the variability due to estimating the parameters $[33,34]$ and the joint model estimate is more robust. We have estimated the parameters using a Bayesian method. The advantage of a Bayesian approach is that it provides exact inferences, versus the asymptotic approximations on which maximum likelihood estimation is based. Joint models improve sensitivity and specificity for predicting anxiety disorder. The AUC from the joint model is higher than that from the two-step model. The Bayesian joint model proposed in this paper has a clear advantage over the naïve two-step model. The joint model is more predictive of adult anxiety disorder than a two-step model. Furthermore, the two-step modeling approach to studying the relationship between a binary primary endpoint and the longitudinal response process is inappropriate due to possible bias and loss of efficiency.

Joint modeling approach has attracted considerable research interest and, as a result, a number of statistical modeling and analysis methods have been suggested for analyzing data with application to various fields [12-14,16-19,23,35,36]. Joint modeling will be accepted commonly for mental health research in the future because it reduces bias and improves efficiency in parameter estimations with the advent of faster computers and the assistance of excellent programmers. The joint model proposed here can be applied to analysis of other psychological and psychiatric datasets where there are longitudinal data first then a binary outcome measure and the question of interest is the association between the development of longitudinal predictor and the binary outcome later. The methodology also can be directly extended to other cases, such as joint modeling of binary outcome with trajectory analysis, factor analysis, and longitudinal repeated measures with informative dropout. 


\section{Competing interests}

The authors declare that they have no competing interests.

Authors' contributions

\begin{tabular}{|l|c|c|c|}
\hline Authors' contributions & HC & YH & NZ \\
\hline Research concept and design & $\checkmark$ & -- & -- \\
\hline Collection and/or assembly of data & $\checkmark$ & -- & -- \\
\hline Data analysis and interpretation & $\checkmark$ & $\checkmark$ & $\checkmark$ \\
\hline Writing the article & $\checkmark$ & $\checkmark$ & $\checkmark$ \\
\hline Critical revision of the article & $\checkmark$ & -- & -- \\
\hline Final approval of article & $\checkmark$ & $\checkmark$ & $\checkmark$ \\
\hline Statistical analysis & $\checkmark$ & $\checkmark$ & $\checkmark$ \\
\hline
\end{tabular}

Publication history

EIC: Jimmt T. Efird, East Carolina University, USA.

Received: 20-Apr-2015 Final Revised: 15-May-2015

Accepted: 20-May-2015 Published: 27-May-2015

\section{References}

1. Cohen P, Kasen S, Chen H, Hartmark C and Gordon K. Variations in patterns of developmental transitions in the emerging adulthood period. Dev Psychol. 2003; 39:657-69. I Article I PubMed

2. Erol RY and Orth U. Self-esteem development from age 14 to 30 years: a longitudinal study. J Pers Soc Psychol. 2011; 101:607-19. | Article | PubMed

3. Robins $R$ and Trzesniewski K. Self-esteem development across the lifespan. Curr Dir Psychol Sci. 2005; 14:158-162. | Article

4. Wallis D. Depression, anxiety and self-esteem: A clinical field study Behav Change. 2002; 19:112-120. I Article

5. Aarons GA, Monn AR, Leslie LK, Garland AF, Lugo L, Hough RL and Brown SA. Association between mental and physical health problems in highrisk adolescents: a longitudinal study. J Adolesc Health. 2008; 43:260-7. | Article | PubMed Abstract | PubMed Full Text

6. Andrews $G$, Crino $R$, Hunt $C$, Lampe $L$ and Page $A$. The treatment of anxiety disorders: Clinician's guide and patient manuals. Cambridge: Cambridge University Press. 1994.

7. Ehntholt KA, Salkovskis PM and Rimes KA. Obsessive-compulsive disorder, anxiety disorders, and self-esteem: an exploratory study. Behav Res Ther. 1999; 37:771-81. | Article | PubMed

8. Marchand A, Goupil G, Trudel G and Belanger L. Fear and social selfesteem in individuals suffering from panic disorder with agoraphobia. Scandinavian Journal of Behaviour Therapy. 1995; 23:163-170. | Article

9. Kogan LS, Smith J and Jenkins S. Ecological validity of indicator data as predictors of survey findings. Journal of Social Service. Research. 1977; 1:117-132. | Article

10. Berenson K, Crawford T, Cohen P and Brook J. The role of parental identification and parents' acceptance in adolescent and young adult self-esteem. Self and Identity. 2005; 4:289-301. I Article

11. First MB, Spitzer RL, Gibbon M and Williams JBW. Structured Clinical Interview for DSM-IV Axis I Disorders, Non-patient Version (SCID-IVNP). New York State Psychiatric Institute, New York, NY. 2000.

12. De Gruttola $V$ and Tu XM. Modelling progression of CD4-lymphocyte count and its relationship to survival time. Biometrics. 1994; 50:100314. | Article | PubMed

13. Faucett $C L$ and Thomas DC. Simultaneously modelling censored survival data and repeatedly measured covariates: a Gibbs sampling approach. Stat Med. 1996; 15:1663-85. I Article I PubMed

14. Huang $Y$, Dagne $G$ and Wu L. Bayesian inference on joint models of HIV dynamics for time-to-event and longitudinal data with skewness and covariate measurement errors. Stat Med. 2011; 30:2930-46. | Article | PubMed

15. Ibrahim JG, Chu H and Chen LM. Basic concepts and methods for joint models of longitudinal and survival data. J Clin Oncol. 2010; 28:2796-
801. | Article | PubMed

16. Song X, Davidian M and Tsiatis AA. An estimator for the proportional hazards model with multiple longitudinal covariates measured with error. Biostatistics. 2002; 3:511-28. | Article | PubMed

17. Wang $Y$ and Taylor JMG. Joint modeling of longitudinal and event time data with application to acquired immunodeficiency syndrome. J Am Stat Assoc. 2001; 96:895-905. | Article

18. Wulfsohn MS and Tsiatis AA. A joint model for survival and longitudinal data measured with error. Biometrics. 1997; 53:330-9. | Article | PubMed

19. Xu J and Zeger SL. Joint analysis of longitudinal data comprising repeated measures and times to events. App/ Stat. 2001; 50:375-387. Article

20. Ratcliffe SJ, Guo W and Ten Have TR. Joint modeling of longitudinal and survival data via a common frailty. Biometrics. 2004; 60:892-9. | Article I PubMed

21. Lunn DJ, Thomas A, Best N and Spiegelhalter D. WinBUGS - a Bayesian modeling framework: concepts, structure, an extensibility. Statistics and Computing. 2000; 10:325-337. | Article

22. Gelman A, Carlin JB, Stern HS and Rubin DB. Bayesian Data Analysis. London: Chapman and Hall. 2003. I Book

23. Tsiatis AA, DeGruttola $V$ and Wulfsohn MS. Modeling the relationship of survival to longitudinal data measured with error: Applications to survival and CD4 counts in patients with AIDS. 1995; 90:27-37. | Article

24. Higgins M, Davidian M and Gilitinan DM. A two-step approach to measurement error in time-dependent covariates in nonlinear mixedeffects models, with application to IGF-I pharmacokinetics. J Am Stat Assoc. 1997; 92:436-448. | Article

25. Ye W, Lin X and Taylor JM. Semiparametric modeling of longitudinal measurements and time-to-event data--a two-stage regression calibration approach. Biometrics. 2008; 64:1238-46. | Article | PubMed

26. Perrin MA, Chen H, Sandberg DE, Malaspina D and Brown AS. Growth trajectory during early life and risk of adult schizophrenia. $\mathrm{Br} J$ Psychiatry. 2007; 191:512-20. | Article | PubMed Abstract | PubMed Full Text

27. Kasen S, Chen H, Sneed JR and Cohen P. Earlier stress exposure and subsequent major depression in aging women. Int J Geriatr Psychiatry. 2010; 25:91-9. | Article | PubMed

28. 28. Kokko K, Nagin DS and Vitaro F. Trajectories of prosocial behavior and physical aggression in middle childhood: Links to adolescent school dropout and physical violence. Journal of Research on Adolescence. 2006; 16:403-428. I Article

29. Piquero AR, Farrington D, Nagin DS and Moffitt TE. Trajectories of offending and their relation to life failure in late middle age: Findings from the Cambridge study in delinquent development. Journal of Research in crime and Delinquency. 2010; 47:151-173. | Article

30. Dick DM, Latendresse SJ, Lansford JE, Budde JP, Goate A, Dodge KA, Pettit GS and Bates JE. Role of GABRA2 in trajectories of externalizing behavior across development and evidence of moderation by parental monitoring. Arch Gen Psychiatry. 2009; 66:649-57. | Article I PubMed Abstract | PubMed Full Text

31. Albert PS and Shih JH. On estimating the relationship between longitudinal measurements and time-to-event data using a simple twostage procedure. Biometrics. 2010; 66:983-7. I Article | PubMed

32. Tsiatis AA and Davidian M. Joint modeling of longitudinal and time-toevent data: an overview. Statistica Sinica. 2004; 14:809-834. I Book

33. Li E, Zhang D and Davidian M. Conditional estimation for generalized linear models when covariates are subject-specific parameters in a mixed model for longitudinal measurements. Biometrics. 2004; 60:1-7. Article | PubMed Abstract | PubMed Full Text

34. Wang CY, Wang N and Wang S. Regression analysis when covariates are regression parameters of a random effects model for observed longitudinal measurements. Biometrics. 2000; 56:487-95. | Article | PubMed

35. Henderson R, Diggle P and Dobson A. Joint modeling of longitudinal 
Chen et al. Journal of Medical Statistics and Informatics 2015, http://www.hoajonline.com/journals/pdf/2053-7662-3-1.pdf

measurements and event time data. Biostatistics. 2000; 1:465-480. | Article

36. Lin H, Turnbull BW, McCulloch CE and Slate EH. Latent class models for joint analysis of longitudinal biomarker and event process data: application to longitudinal prostate-specific antigen readings and prostate cancer. J Am Stat Assoc. 2002; 96:895-905. | Article

\section{Citation:}

Chen $\mathrm{H}$, Huang $\mathrm{Y}$ and Zhang N. Joint modeling of a linear mixed effects model for selfesteem from mean ages 13 to 22 and a generalized linear model for anxiety disorder at mean age 33. J Med Stat Inform. 2015; 3:1. http://dx.doi.org/10.7243/2053-7662-3-1 